\title{
Spin-polarized transport through carbon nanotubes
}

\author{
S. Krompiewski* \\ Institute of Molecular Physics, Polish Academy of Sciences, ul. M. Smoluchowskiego 17, 60-179 Poznań, \\ Poland
}

Received zzz, revised October 30, 2004, accepted zzz

Published online zzz

PACS 85.35.Kt, 85.75.-d, 81.07.De, 73.63.-b

\begin{abstract}
Carbon nanotubes (CNT) belong to the most promising new materials which can in the near future revolutionize the conventional electronics. When sandwiched between ferromagnetic electrodes, the CNT behaves like a spacer in conventional spin-valves, leading quite often to a considerable giant magneto-resistance effect (GMR). This paper is devoted to reviewing some topics related to electron correlations in CNT. The main attention however is directed to the following effects essential for electron transport through nanotubes: (i) nanotube/electrode coupling and (ii) inter-tube interactions. It is shown that these effects may account for some recent experimental reports on GMR, including those on negative (inverse) GMR.
\end{abstract}

Copyright line will be provided by the publisher

1 Introduction A particularly challenging class of materials for the future electronics are carbon nanotubes, i.e. graphite sheets rolled up in such a manner that they form very long and thin (in atomic scale) cylinders. Their length can be of the order of several micrometers whereas typical cross-sections of single wall carbon nanotubes (SWCNT) are a few nanometers in diameter, and roughly up to 10 times more for multi-wall carbon nanotubes (MWCNT) . Both the SWCNTs and MWCNTs have been attracting much attention of many researchers since they were discovered [1].

It is now clear that the electronics based on conventional semiconducting devices (e.g. silicon technology) has faced fundamental limitations as far as further miniaturization is concerned. The reasons are various, including the fact that the relative amount of surface states - destructive for electronic transport properties - increases rapidly in 2- and 3-dimensional systems when their sizes are being reduced. Likewise, it is predicted that the $\mathrm{SiO}_{2}$ layer in the MOSFET will be too thin soon (within 10 years or so) to retain its insulating properties [2]. So, there is much interest of physicists, technologists and engineers in new generation nano-scale devices, including molecular ones. The adopted approach is the so-called bottom up philosophy, aiming at designing electronic devices at the molecular level. In contrast to siliconbased devices, the molecular ones (incl. carbon nanotubes, CNT) can successfully cope with the more and more demanding miniaturization requirements without worsening their multi-functional properties. Most probably the CNTs are also good candidates for spintronics applications in view of their unusually long spin diffusion length. It is well-known that electrons can travel through carbon nanotubes up to many hundreds of nanometers, still keeping their momentum and spin orientation. So CNTs are believed to be useful in the near future microelectronics (or molecular electronics) as interconnects, and possibly also as active elements in integrated circuits. Physical properties of the CNTs connected to metallic electrodes depend critically on a quality of CNT/metal junctions, and may evolve with increasing transparencies of the junctions: from the Coulomb blockade regime, through the Kondo effect, and Fabry-Perot resonator-like behavior up to the Fano resonance.

This paper is organized as follows. First, some rather well-known problems concerning electron correlation regimes in CNTs are recalled (sub-sections 2.1 - 2.4). Second, a short survey of selected papers,

* Corresponding author: e-mail: stefan@ifmpan.poznan.pl, Phone: +48 618695 126, Fax: +48 618684524 
devoted to the problem of both the CNT-electrode coupling (Sec. 3) as well as the GMR effect (Sec. 4) will be made. Finally a tight-binding approach combined with the Green's function method will be described and applied to the coherent spin-polarized electronic transport through the CNTs.

\section{Transport regimes}

2.1 Ballistic regime Well-contacted long SWCNTs are essentially one dimensional systems from the point of view of their transport properties, since they can be viewed as translational in the axial direction and quantized in the transverse one. It was shown that in such a case they behave like a Fabry-Perot resonator, revealing spectacular $d I / d V-V-V_{g}$ (differential-conductance vs. bias and gate voltage) contour plots [3 4]. The relevant energy scale here is the energy level spacing $\Delta=h v_{F} /(2 L)$, which determines the electron wavelength and is directly related to multiple reflections and superpositions corresponding to the round trip length $2 \mathrm{~L}$ of electronic waves, where $\mathrm{L}$ and $v_{F}$ stand for the SWCNT length, and the Fermi velocity, respectively.

2.2 Coulomb Blockade The opposite transport regime is the Coulomb blockade one. It takes place at low temperatures when the CNT is weakly coupled to the electrode and may be regarded as a quantum dot. The relevant energy scale in this case is the so-called addition energy $\left(E_{\text {add }}\right)$ equal to the inter-level energy spacing ( $\Delta E=\Delta / 2$ due to lifting of the band degeneracy) plus the charging energy $E_{c}=e^{2} / C$, where $C=C_{s}+C_{d}+C_{g}$ is the total capacitance between the dot and the source, drain and gate. In order for the effect to be pronounced, $E_{c}$ must be greater than $\Delta E$, and the junction resistances should be greater than the inverse of the conductance quantum $\left(R>h / e^{2}\right)$. The set of equations describing the Coulomb blockade reads [5]:

$$
\begin{aligned}
& U(N)=\left[-e\left(N-N_{0}\right)-C_{g} V_{g}\right]^{2} / 2 C, \quad F(N)=U(N)+\sum_{i=1}^{N} E_{i}, \\
& \mu_{\text {dot }}(N)=F(N)-F(N-1), \quad E_{a d d}=\mu_{\text {dot }}(N+1)-\mu_{\text {dot }}(N)=E_{c}+\Delta E,
\end{aligned}
$$

where $U(N)$ is the electrostatic energy in the presence of $N-N_{0}$ excess electrons and the gate voltage $V_{g}, \mathrm{~F}(\mathrm{~N})$ is the total energy, and $\mu_{d o t}$ and $E_{i}$ are the chemical potential and the energy levels of the dot, respectively. In general the addition energy gap prevents electrons from jumping to and off the dot. The situation changes when, due to the gate voltage, the unoccupied energy level of the dot aligns with the chemical potentials of the contacts (falls into the transport window). The electrons can then be pumped one by one from source to drain. Such a process is called sequential tunnelling. There is a simple rule of thumb to evaluate $E_{c}$ and $\Delta E$ for SWCNT, namely $E_{c} \sim 5 \mathrm{meV} / \mathrm{L}[\mu \mathrm{m}]$ and $\Delta E \sim 1 \mathrm{meV} / \mathrm{L}[\mu \mathrm{m}]$. So the ratio $E_{c} / \Delta E$ is roughly equal to 5 and it does not depend on the length. The simplest way of energy shell filling in SWCNT is the 2-fold one (even-odd). Adding an electron to the occupied level costs just $E_{c}$ energy, whereas the energy required to add another electron is increased by what is needed to reach the successive energy level and amounts to $E_{c}+\Delta E$. In some SWCNTs the even-odd shell filling was experimentally established [6], whereas in others the four-fold shell filling was reported [7].

2.3 Kondo effect It turns out that if the dot/electrodes coupling is not too poor, a higher order tunnelling process can overcome the Coulomb blockade. It is called the co-tunnelling and may be thought of as a correlated jump of an electron from the dot to the drain when another electron jumps on the dot from the source at the same time. In the Kondo regime the crucial role is played by processes for which the entering electron and the leaving one have opposite spins. The net effect then is as if the quantum dot flipped 
its spin, very much like in the conventional (magnetic impurity) Kondo effect with resonance scattering of conduction electrons by localized magnetic moments. In the former case however resonance conductance rather than resonance scattering takes place, and consequently the conductance increases when temperature is lowered from the Kondo temperature to zero in contrast to the aforementioned conventional case. Experimentally, the Kondo effect is nicely seen in the $d I / d V-V-V_{g}$ (diamond) plots, as clear nonzero conductance horizontal features, in the vicinity of $\mathrm{V}=0$, within the diamonds corresponding to odd numbers of electrons [8].

2.4 Luttinger liquid One-dimensional systems with strong electron correlations do not behave like the Fermi-liquid with free electron-like low-energy excitations (quasi-particles). Instead, electrons form a correlated state called the Luttinger liquid (LL) with plasmon-like excitations. Metallic carbon nanotubes belong to this class of materials under certain circumstances, and they represent the best realization of the LL physics [9]. The strength of electron-electron interactions is described by the Luttinger parameter $g=1 / \sqrt{\left(1+2 E_{c} / \Delta E\right)}$, which is less than 1 for repulsive Coulomb interactions and equals 1 in the absence of interactions. The parameter $g$ is just the ratio of $v_{F}$ over $v_{g}$, with the latter being the plasmon velocity. Incidently, the $v_{g}$-s for spin plasmons and charge plasmons are different, but no spin charge separation has ever been experimentally established in SWCNTs, to our knowledge. The interactions critically influence electronic transport, because the tunnelling of electrons from a metal electrode (Fermi liquid) to the SWCNT (LL) is only possible if the tunnelling electrons have energies high enough to couple with the plasmon modes in the LL. This is in contrast to the tunnelling between two Fermi liquids, where no energy dependence is basically expected. Consequently, LL systems show power-law temperature- and bias-dependence of the conductance $\mathcal{G} \sim T^{\eta}, \quad d I / d V \sim V^{\eta}$, for small and large $V$, respectively. Moreover it can be shown that the scaled differential conductance $\frac{d I}{d V} / T^{\eta}$ is a universal function of $e V / k_{B} T$.

3 Some experimental aspects It is a usual practice to select carbon nanotubes for further transport measurements on the basis of their room temperature two-terminal conductance $\mathcal{G}_{\mathcal{R}} \mathcal{T}$. Tubes with conductances almost independent of gate voltage are classified as metallic, others - as semiconducting. It is convenient to define a transmission probability of contacts as $P_{c}=2 /\left(1+\mathcal{G}_{\max } / \mathcal{G}_{R T}\right)$, where $\mathcal{G}_{\max }=4 e^{2} / \mathrm{h}$ is the maximum possible conductance of a SWCNT $\left(0 \leq P_{c} \leq 1\right)$ [10]. At low temperature, for lowtransparency samples $\left(P_{c} \sim 0.15\right)$, Coulomb blockade sets in, with periodic sharp peaks separated by zero-conductance valleys in the $\mathcal{G}$ vs. $V_{g}$ plots. For better transparencies $\left(P_{c} \sim 0.6\right)$ the Kondo regime is reached. Eventually, for $P_{c} \sim 0.9$ one enters a ballistic regime, characterized by the so-called inverse diamond structures with maxima of the conductance near the middle of the diamonds. It means that very well-contacted SWCNT act as etalon-like resonant cavities with the (inverted) diamond structue period varying as $1 / L$, which lends support to the view that open-contact SWCNT are ballistic phase coherent wires. Quite recently a series of experimental papers has appeared which shows that nanotubes wellcontacted to metal electrodes can now be fabricated in a controllable way ([11], [12]). The best transparent interfaces are for Pd and Au electrodes. In some cases the transparency is considerably improved upon annealing (e.g. for Ti electrodes). As shown in [13] it is even possible to observe practically all the transport regimes together on the same sample by just changing gate voltage. It means that gate voltage may strongly influence the dot/electrode tunnelling coupling.

4 GMR in carbon nanotubes The giant magnetoresistance (GMR) effect in CNTs was first measured 5 years ago [14], it was estimated that the spin diffusion length of electrons flowing through a tube is ca. $130 \mathrm{~nm}$ or most probably more than that. Bearing in mind, that nowadays CNT lengths used in electric current measurements, are quite often as short as $200-250 \mathrm{~nm}$, it means that practically electrons travel through nanotubes in a spin-coherent way (no spin flips). Experimental papers concern mostly MWCNTs electrically contacted by cobalt [14, 15], but there are also reports on iron [16] and permalloy [17] 
contacted multi-walled and single wall carbon tubes. The results depend very strongly on ferromagneticelectrode/CNT interfaces, yielding the net GMR effect of the order of $10-40 \%$. It should be stressed however that also inverse GMR has been reported of roughly similar magnitude but with negative sign [15] 17]. A very surprising data concerning Fe-contacted SWCNT were reported in [16], where a measured GMR effect approached $100 \%$, i.e. transport was completely blocked in the antiparallel aligned configuration. It seems that so far a consensus has been gained that the main contribution to the spindependent transport comes from a ferromagnet/CNT junction and a CNT itself acts only as a quasi ballistic waveguide. The junction properties are typically temperature, magnetic field and gate voltage dependent. There are suggestions that at the interface an antiferromagnetic (e.g. CoO) layer may be formed, such a layer would be undoubtedly temperature and magnetic field sensitive, and could also be responsible for hysteretic (magnetic field sweeping direction) effects. In [18] it was shown theoretically that an additional magnetic layer at the interface does really influence the GMR effect very substantially, and can even block the current in the antiparallel configuration. It is also quite possible that local magnetic domains which touch the carbon nanotube might be misaligned with respect to the total electrode magnetization. Other important points concern the internal structure of MWCNT, i.e first of all (i) the inter-tube interactions, and (ii) whether or not the particular tubes are in (out of) contact with the electrodes. These problems will be addressed in the following.

5 Theoretical approach The studies are carried out within the framework of the single-band tightbinding model for $p_{z}(\pi)$ electrons in the carbon nanotubes. The magnetic electrodes are spin-split by assuming spin-dependent on-site potentials therein. The Green's function technique has been used, along the line of the non-equilibrium transport formalism, and under constraint of global charge neutrality. The so-called extended molecule concept is adapted, by incorporating to the central part of the system not only the entire molecule (CNT) but also two closest magnetic atomic planes from the left- and right-hand side electrodes [20]. The Green's function is defined as $G=\left(\hat{1} E-H_{\mathrm{C}}-\Sigma_{\mathrm{L}}-\Sigma_{\mathrm{R}}\right)^{-1}$, whereas the density matrix of electrons and current (per spin) are given by:

$$
\begin{aligned}
& n=\frac{1}{2 \pi} \int d E G\left[f_{L} \Gamma_{L}+f_{R} \Gamma_{R}\right] G^{\dagger}, \\
& I=\frac{e}{h} \int_{-\infty}^{\infty} d E\left(f_{L}-f_{R}\right) \operatorname{Tr}\left[\Gamma_{L} G \Gamma_{R} G^{\dagger}\right] .
\end{aligned}
$$

At low bias and temperature the latter leads to the conductance $\mathcal{G}=\frac{e^{2}}{h} \operatorname{Tr}\left[\Gamma_{L} G \Gamma_{R} G^{\dagger}\right]$, where $\alpha=\mathrm{L}, \mathrm{R}, \mathrm{C}$ refer to the left, right electrodes and the extended molecule, respectively. Moreover $\Gamma_{\alpha}=$ $i\left(\Sigma_{\alpha}-\Sigma_{\alpha}^{\dagger}\right), \quad \Sigma_{\alpha}=V_{\mathrm{C}, \alpha} g_{\alpha} V_{\mathrm{C}, \alpha}^{\dagger}$, where $V_{L(R), C}$ describes the coupling between the electrodes and the extended molecule, and $f_{\alpha}$ is the Fermi-Dirac distribution function. The $g_{\alpha}$ is the $\alpha$-th electrode surface Green's function. The latter has been calculated as in [21], but summed over the 2-dimensional Brillouin zone (while Fourier transforming back to the real space) by the special-k-points method [22]. The "pessimistic" definition is used $G M R=\left(\mathcal{G}_{\uparrow, \uparrow}-\mathcal{G}_{\uparrow, \downarrow}\right) / \mathcal{G}_{\uparrow, \uparrow}$, where the arrows indicate the relative magnetization alignments of the left and right external electrodes. The inter-tube hopping parameters are taken after [23] as

$$
t_{i n t}=-(t / 8) \sum_{l, j} \cos \theta_{l j} e^{\frac{d_{l j}-b}{\delta}}
$$

where $\theta$ is the angle between the $\pi$ orbitals, $\mathrm{d}$ is a relative distance, $t$ stands for the nearest neighbor intra-tube C-C hopping integral (chosen as energy unit), $\delta=0.45 \AA$ and $\mathrm{b}=3.34 \AA$. 
6 Modelling of the devices and the results The contacts are modelled as bulk fcc-(111) slabs infinite in all the 3 directions and connected to the CNT through a neck composed of two finite atomic planes. In my previous papers, very special geometries of the SWCNT $(6,6)$ armchair and the double-walled CNT $(2,2) @(6,6)$ were studied (see [18] - [20] for details). In those cases it was possible to construct the end-contacted devices in such a way that interface carbon atoms were placed exactly above the centers of equilateral triangles formed by the adjacent transition metal atoms. Now this construction has been generalized so that in principle any CNT may be end-contacted, by performing a relaxation under a given potential, e.g. Lennard-Jones' (see Fig. 1). The procedure goes as follows: (i) nanotubes are generated using the codes A-2 and A-3 of [24], (ii) the tubes are placed vertically on the fcc-(111) transition metal contacts, (iii) the outer tube is kept fixed while the electrodes and the inner tube are free to rotate and move along the axial direction. The relaxation is made by the exact numerical minimization of energy of the device, assuming that energetically most favorable positions for interface $\mathrm{C}$ atoms would be again those, where they had exactly 3 transition metal atom nearest neighbors.
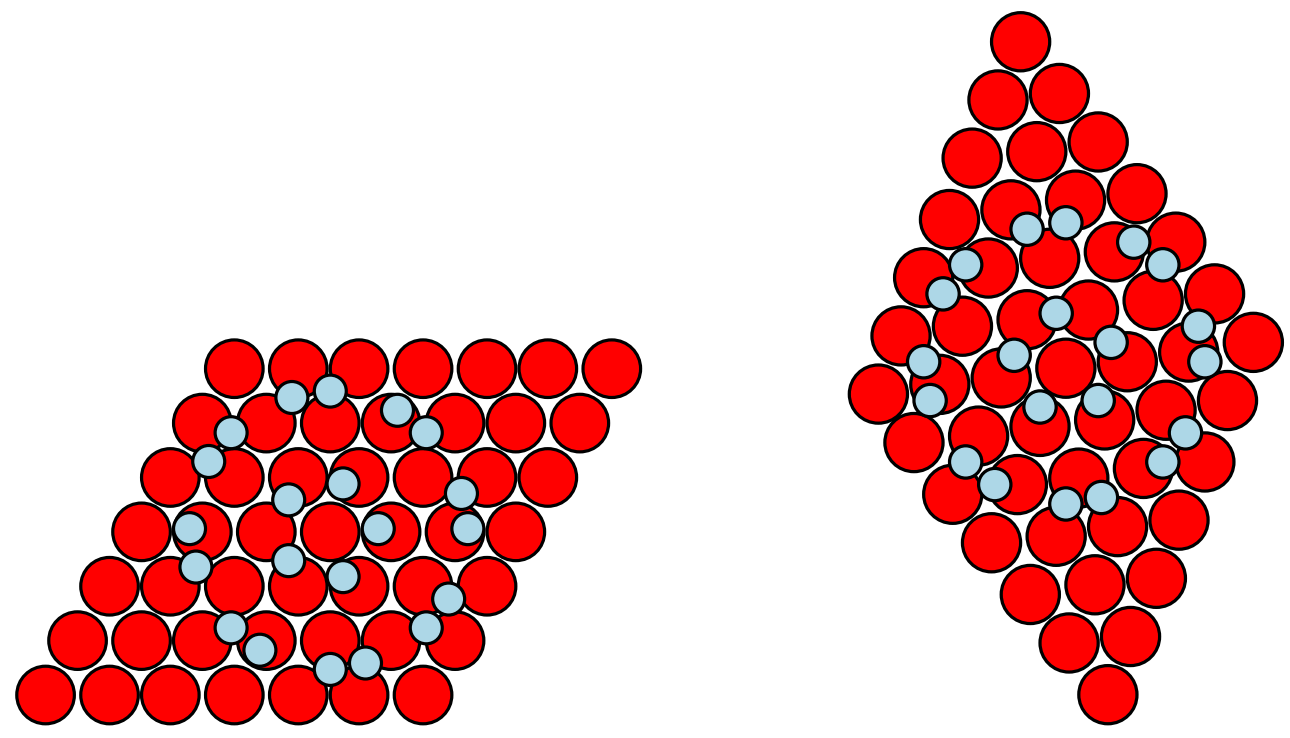

Fig. 1 One of the interfaces between the DWCNT $(5,0) @(8,8)$ (small spheres) and the fcc(111) metal electrode (big spheres) before (left hand side) and after (r.h.s) the relaxation.

In Fig. 2 conductances are presented for parallel (PA - thick solid line) and antiparallel (AP - thick dotted line) alignments of magnetic electrodes, along with the corresponding GMRs (thin line). The figure shows results for a double-walled carbon nanotube (DWCNT) composed of the outer armchair $(8,8)$ tube and the inner zigzag $(5,0)$ one. In order to keep the length of both the tubes close to each other there are 45 and 39 carbon rings for the inner and outer tubes, respectively. As readily seen the GMR in the charge neutrality point $(E=0)$ is above $20 \%$ and happens to be rather robust against small energy changes. Judging from the conductances the presented results correspond to the low resistivity junctions. The relevant energy scale is related to inter-level spacing $\Delta E \sim 0.15$ in the adopted units $(|t|$ and the graphene lattice constant). There are theoretical and experimental arguments that such a fine structure gets washed out if there is disorder, e.g. due to interactions with incommensurate inner tubes, structural imperfections or impurities [25]. Figure 2 (b) shows the conductances after averaging over the energy interval equal to $\Delta E$, and the resulting GMR.

To gain a deeper insight into the effect of the inner tube on the overall conductance of the device, Fig. 3 presents the results for the case when both the tubes are of armchair-type, but the inner one is by one carbon-ring shorter, being thereby out of contact to the drain. It is easily seen that now the GMR is quite 

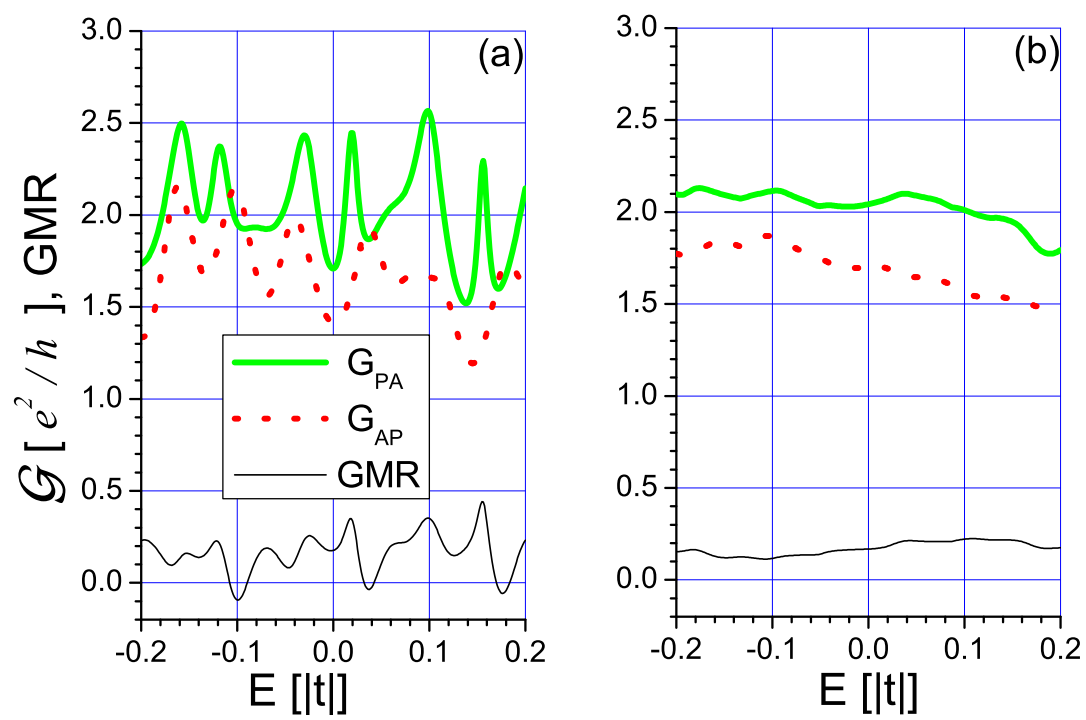

Fig. 2 (a): Conductance and GMR for a double-walled carbon nanotube, which consists of 45 zigzag rings and 39 armchair rings $(45-(5,0) @ 39-(8,8))$. (b): The energy averaged conductances over energy bins equal to the energy-level spacing, and the resulting GMR. The averaging mimics the anticipated effect of disorder

unstable with respect to energy changes near $E_{F}=0$. On the other hand however, the GMR gets robust and positive if either the inner-tube interaction vanishes (Fog. 3 (b)) or there are the above mentioned disorder-induced self-averaging mechanisms. The present results generalize those of [20], obtained for different nanotube diameters and perfect geometical matching at the interfaces. The GMR of the DWCNT is basically positive, if a wide-spread opinion that the current flows exclusively through the outer nanotube
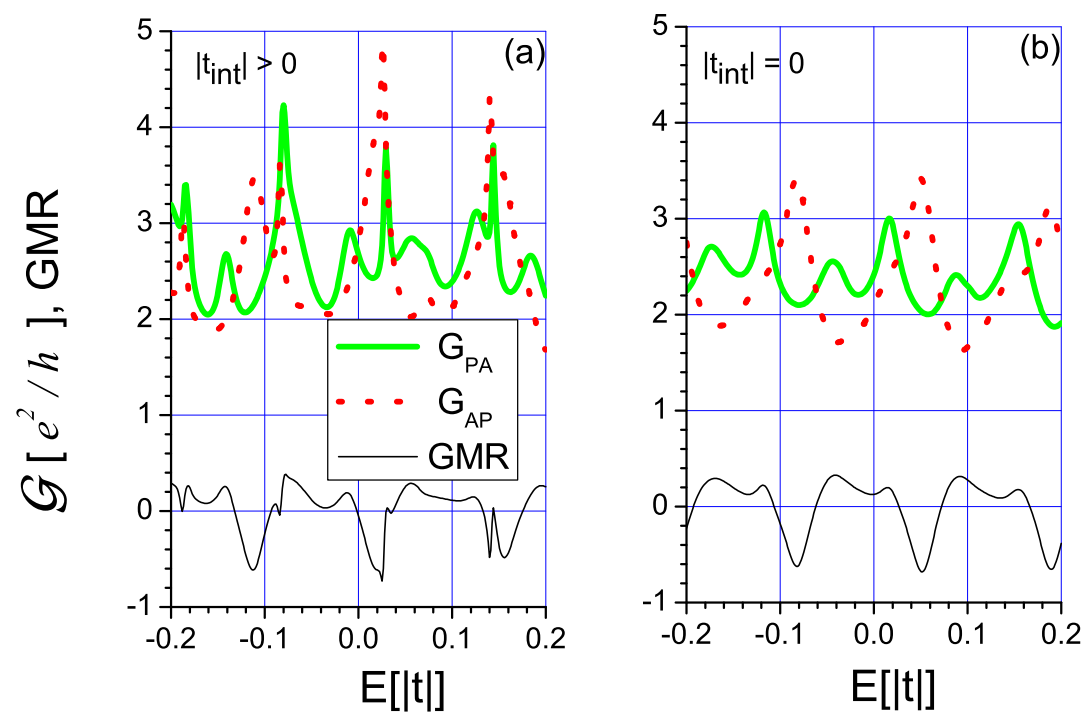

Fig. 3 (a): Conductances and GMR for the armchair DWCNT $(3,3) @(8,8)$. There are $39(38)$ carbon rings of the outer (inner) tube, so that the inner tube is out of contact with the drain. (b): Same as in (a) but with no inter-tube interactions. 
is accepted. The new observation is that even in this case the inverse GMR may be encountered in the vicinity of $E_{F}$. The inter-tube interactions are quite decisive for electronic transport in the DWCNT even if the inner tube is not fully contacted by the electrodes, provided it is metallic (of the armchair-type). Note that the maximum conductance in Fig. 3 (a) may exceed $4 e^{2} / h$ in contrast to the cases presented in Figs. 2 (a) and 3 (b). This means that in the former case the inner tube opens additional conduction channels for electric transport. Another observation emerging from the present studies is that the inverse GMR is more likely to occur in systems with a small amount of disorder, where energy-level features do not get smeared out.

7 Summary The spin-dependent transport through carbon nanotubes electrically contacted by metal electrodes has been addressed. The main transport regimes, resulting from electron-electron interactions have been discussed and shown to be strictly related to the nanotube/electrode junction quality. A method to model CNT/metal interfaces (end-contacted devices) has been presented and exemplified by two types of DWCNTs defined in such a way that the current flows through the outer tube only. It has been shown that for high-transparency interfaces the GMR effect is basically positive, the inverse GMR may however be also encountered in this limit. The latter comes about only on the inter enegy-level spacing scale, when due to different boundary conditions, the conductance has a maximum at the charge neutrality point for the antiparallel configuration and a reduced value for the parallel alignment. The probability of such a coincidence is increased by the fact that on symmetry grounds the conductance peaks are more strongly spin-split in the parallel configuration than in the antiparallel one.

Acknowledgements I am grateful to Giovanni Cuniberti for fruitful discussions. The KBN (research project PBZKBN-044/P03-2001) as well as the support by the Centre of Excellence for Magnetic and Molecular Materials for Future Electronics, within the European Commission contract No. G5MA-CT-2002-04049, are also acknowledged.

\section{References}

[1] S. Ijima, Nature (London), 354, 56 (1991).

[2] M. Schulz, Nature (London), 399, 729 (1999).

[3] W. Liang, M. Bockrath, D. Bozovic, J. H. Hafner, M. Tinkham, and H. Park, Nature (London) 411, 665 (2001).

[4] S. Krompiewski, J. Martinek, J. Barnaś, Phys. Rev. B, 66, 073412 (2002).

[5] L. P. Kouwenhoven, D. G. Austing, and S. Tarucha, Rep. Prog. Phys., 64, 701 (2001).

[6] D. H. Cobden and J. Nygård, Phys Rev. Lett, 89, 046803 (2002).

[7] W. Liang, M. Bockrath, and H. Park, Phys Rev. Lett, 88, 126801 (2002).

[8] J. Nygård, D. H. Cobden, and P. E. Lindelof, Nature (London) 408, 342 (2000).

[9] M. Bockrath, D. H. Cobden, J. Lu, A. G. Rinzler, R. E. Smalley, L. Balents, and P, L. McEuen, Nature (London) 397, 598 (1999).

[10] J. Nygård, D. H. Cobden, cond-mat/0105289

[11] A. Javey, J. Guo, Q. Wang, M. Lundstrom, and H. Dai, Nature (London) 424, 654 (2003).

[12] B. Babić, J. Furer, M. Iqbal, and C. Schönenberger, cond-mat/0406626

[13] B. Babić and C. Schönenberger, cond-mat/0406571

[14] K. Tsukagoshi,B.A. Alphenaar, and H. Ago, Nature (London), 401, 572 (1999).

[15] B. Zhao, I. Mönch, T. Mühl, and C.M. Schneider, Appl. Phys. Lett. 80, 3144 (2002).

[16] A. Jensen, J. Nygård, and J. Borggreen, in Toward the controllable quantum states, Proceedings of the International Symposium on Mesoscopic Superconductivity and Spintronics, H. Takayanagi and J. Nitta (eds.), pp. 33-37, World Scientific (2003).

[17] J. Kim, J-R. Kim, J.W. Park, J-J. Kim, K. Kang, N. Kim, B-C. Woo, Physica E, 18, 208 (2003).

[18] S. Krompiewski, J. Phys.: Condens. Matter 16, 2981 (2004).

[19] S. Krompiewski, J. Magn. Magn. Mat. 1645, 272 (2004).

[20] S. Krompiewski, R. Gutierrez, G. Cuniberti, Phys Rev. B, 69, 155423 (2004).

[21] T.N. Todrov et al., J. Phys.: Cond. Mat. 5, 2389 (1993)

[22] S.L. Cunningham, Phys. Rev. B 10, 4988 (1974)

[23] S. Roche, F. Triozon, A. Rubio, and D. Mayou, Phys. Rev. B 64, 121401 (2001).

[24] R. Saito, M. S. Dresselhaus, and G. Dresselhaus, Physical Properties of Carbon Nanotubes, Imperial College Press (1998)

[25] F. Triozon, S. Roche, A. Rubio, and D. Mayou, Phys Rev. B, 69, 121410 (2004). 